\title{
On Matrix Fourier Multipliers for Semi-orthonormal Binary Framelets and Perturbation of Gabor Frames and Applications
}

\author{
Qingjiang Chen, Bingzhe Wei, Yanbo Zhang
}

\author{
School of Science, Xi'an University of Architecture and Technology, Xi'an 710055, China \\ qjchen66xytu@126.com
}

\begin{abstract}
Keywords: Bivariate Gabor frames, dual Gabor frames, Banach space, time-frequency analysis, wavelet transfers, filter banks, paraunitary operator

Abstract. Mechanical engineering is the profession in which knowledge of the mathematical and natural sciences. The concept for matrix Fourier multipliers concerning bivariate tight multi-wavelet frames is introduced in this paper. Based on matrix theory, a sufficient and necessary condition for a matrix-valued function, which becomes matrix Fourier multipliers for bivariate tight multi-wavelet frames is provided. The existence of bivariate Gabor frames with compact support is discussed. Sufficient conditions for irregular bivariate Gabor system to be frames are presented by means of frame multiresolution analysis and paraunitary vector filter bank theory.
\end{abstract}

\section{Introduction and fundamentals}

The frame theory plays an important role in the modern time-frequency analysis.It has been de -veloped very fast over the last twenty years, especially in the context of wavelets and Gabor systems. In her celebrated paper[1]. Mechanical engineering is the second largest and one of the oldest disciplines; broadest of all engineering disciplines. Mechanical engineers apply the principles of mechanics and energy to the design of machines and devices: Energy and Motion. Overall foundation: mathema -tics physics, chemistry, biology, analysis skills, communication skills, computation skills. To study some deep problems in nonharmonic Fourier series, Duffin and Schaeffer[2] introdu -ce the notion of frames for a separable Hilbert space in 1952. Basically, Duffin and Schaeffer abs -tracted the fundam -ental notion of Gabor for studying signal processing [3]. These ideas did not se -em to generate much general interest outside of nonharmonic Fourier series however (see Young's [4]) until the landmark paper of Daubechies, Grossmann, and Meyer [5] in 1986. After this ground breaking work, the theory of frames began to be more widely studied both in theory and in applicat -ons [6-8], such as signal processing, image processing, data compression and sampling theory. The notion of frame multiresolution analysis (FMRA) as described by [6] generalizes the notion of multi -resolution analysis (MRA) by allowing non-ex act affine frames. However, subspaccs at different resolutions in a FMRA are still generated by a fra -me formed by translares and dilates of a single biv-ariate function. Inspired by [5] and [7], we intro -duce the norion of a generalized bivariate multiresolution structure (GBMS) of $L^{2}\left(R^{2}\right)$ geneated by several functions of integer translates $L^{2}\left(R^{2}\right)$. We demonstrate that the generalized bivariate multire solution structure has a pyramid decom position scheme and obiain a frame-like decomposition based on such a GBMS. It also lead to new constructions of affine of $L^{2}\left(R^{2}\right)$. Since the majority of informa tion is multidimensional information, many researchers interest themselves in the investigation into bivariate wavelet theory. The classical method for constructing multi-variate wavelets is that separa-ble bivariate wavelets may be obtained by means of the tensor product of some univariate wavelet frames. It is significant to investigate nonseparable multivariate wavelet frames. 
The concept for matrix Fourier multipliers concern-ing bivariate tight multi-wavelet frames is introduced in this paper. Based on matrix theory, a sufficient and necessary condition for a matrix -valued function, which becomes matrix Fourier multipliers for bivariate tight multi-wavelet frames is provided. Lots of constructive examples are also presented.

Let $W$ be a separable Hilbert space and $\Lambda$ is an index set. We recall that a sequence $\left\{\lambda_{v}: v \in Z\right\} \subseteq W$ is a frame for $\mathrm{H}$ if there exist positive real numbers $B, C$ such that

$$
\forall \hbar \in W, \quad B\|\hbar\|^{2} \leq \sum_{v \in \Lambda}\left|\left\langle\hbar, \lambda_{v}\right\rangle\right|^{2} \leq C\|\hbar\|^{2}
$$

A sequence $\left\{\lambda_{v}: v \in Z\right\} \subseteq W$ is a Bessel sequence if (only) the upper inequality of (1) holds. If only for all $\hbar \in \Lambda \subset W$, the upper inequality of (1) holds, the sequence $\left\{\lambda_{v}\right\} \subseteq W$ is a Bessel sequence with respect to (w.r.t.) $\Lambda$. If $\left\{f_{v}\right\}$ is a frame, there exists a dual frame $\left\{f_{v}^{*}\right\}$ such that

$$
\forall \Upsilon \in W, \Upsilon=\sum_{v \in \Lambda}\left\langle\Upsilon, f_{v}\right\rangle f_{v}^{*}=\sum_{v \in \Lambda}\left\langle\Upsilon, f_{v}^{*}\right\rangle f_{v} .
$$

To state our results, the Fourier transform of an function $f(x) \in L^{1}\left(R^{2}\right)$ is defined by

$$
F f(\omega)=\hat{f}(\omega)=\int_{R^{2}} f(x) e^{-2 \pi i x \omega} d x, \quad \omega \in R^{2},
$$

which, as usual, can be naturally extended to functions in $L^{2}\left(R^{2}\right)$. For a sequence $c=\{c(c)\} \in$ $\ell^{2}(z)$, we define its discrete-time Fourier transform as the function in $L^{2}(0,1)^{2}$ by

$$
F c(\omega)=C(\omega)=\sum_{v \in Z^{2}} c(v) e^{-2 \pi i x \omega} d x,
$$

For $s>0$ we denote by $H^{s}\left(R^{2}\right)$ the Sobolev space of all binary functions $h(x) \in H^{s}\left(R^{2}\right)$ such that

$$
\int_{R^{n}}|\hat{h}(\gamma)|\left(1+\|\gamma\|^{2 s}\right) d \gamma<+\infty .
$$

A system $\left\{g_{m, n}\right\}=\left\{T_{n a} E_{m b} g\right\} \quad(m, n \in Z)$ is a Gabor frame or Weyl-Heisenberg frame for $L_{2}(R)$, if there exist two constants $A, B>0$, where $a, b \in R^{+}$are constants such that

$$
A\|\hbar\|^{2} \leq \sum_{m, n \in Z}\left|\left\langle\hbar, g_{m, n}\right\rangle\right|^{2} \leq B\|\hbar\|^{2}
$$

holds for all $\forall \hbar \in L_{2}(R)$ which is a square integrable measur. For a Gabor frame $\left\{g_{m, n}\right\}$ the analysis mapping (also called Gabor transform) $U_{g}$, given by

$$
U_{g}: f \rightarrow\left\{<f, g_{m, n}>\right\}_{m, n}, \forall f \in L_{2}(R)
$$

and its adjoint, the synthesis mapping (also called Gabor expansion) $U_{g}^{*}$, given by

$$
U_{g}^{*}:\left\{c_{m, n}\right\} \rightarrow \sum_{m, n} c_{m, n} g_{m, n}, \quad \forall\left\{c_{m, n}\right\} \in \ell^{2}(Z)
$$

are bounded linear operators. The Gabor frame operator $S_{g}$ is defined by $S_{g}=U_{g}^{*} U_{g}$. Explicitly,

$$
S_{g} f \rightarrow \sum_{m, n}<f, g_{m, n}>g_{m, n}, \quad \forall f \in L_{2}(R)
$$

If $\left\{g_{m, n}\right\}$ forms a Gabor frame for $L_{2}(R)$, then $\forall f \in L_{2}(R)$ can be written as

$$
f=\sum_{m, n}<f, g_{m, n}>h_{m, n}=\sum_{m, n}<f, h_{m, n}>g_{m, n},
$$

where $h_{m, n}$ are the elements of the dual frame, given by $h_{m, n}=S^{-1} g_{m, n}$. Equation (ㅁ) provides a con-structive answer how to recover $f(x)$ from its Gabor transform $\left\{<f, g_{m, n}>\right\}_{m, n \in Z}$ for given analysis window $g(x)$ and how to compute the coefficients in the series expansion $f=\sum_{m, n \in Z} C_{m, n} g_{m, n}$ for given atom $g(x)$. The key is the cor-responding dual frame $\left\{\left\langle f, g_{m, n}\right\rangle\right\}_{m, n}$. A detailed analysis of Gabor frames brings forward some features that are basic for a further understanding of Gabor analysis. Most of these features are not shared by other frames such as wavelet frames. 


\section{Characterization of generalized multiresolution structure}

To characterize such a GMS, we first introduce the concept of an pseudoframes and tight frames.

Definition 1. Let $\left\{T_{v a} f, v \in Z^{2}\right\}$ and $\left\{T_{v a} \widetilde{f}, v \in Z^{2}\right\}$ be two sequences in space $L^{2}\left(R^{2}\right)$. Let $\Omega$ be a closed subspace of space $L^{2}\left(R^{2}\right)$. We say $\left\{T_{v a} f, v \in Z^{2}\right\}$ forms an affine pseudoframe for $\Omega$ with respect to $\left\{T_{v a} \widetilde{f}, v \in Z^{2}\right\}$ if

$$
\forall \Gamma \in \Omega, \Gamma(x)=\sum_{v \in Z^{2}}\left\langle\Gamma, T_{v a} \widetilde{f}\right\rangle T_{v a} f(x)
$$

It is important to note that $f$ and $\widetilde{f}$ need not be contained in $\Omega$. Example 2 is such case. Consequently, the positions of $T_{v a} f$ and $T_{v a} \widetilde{f}$ are not generally commutable [5], i.e.,

$$
\forall \Gamma(x) \in \Omega, \quad \sum_{v \in Z^{2}}\left\langle\Gamma, T_{v a} f\right\rangle T_{v a} \widetilde{f}(x) \neq \sum_{v \in Z^{2}}\left\langle\Gamma, T_{v a} \widetilde{f}\right\rangle T_{v a} f(x)=\Gamma(x) .
$$

Define an analysis operator $K: \Omega \rightarrow \ell^{2}\left(Z^{2}\right)$ by

$$
\forall \Gamma(x) \in \Omega, \quad K \Gamma=\left\{\left\langle\Gamma, T_{v a} f\right\rangle\right\},
$$

and define another operator $L: \ell^{2}(Z) \rightarrow H$ such that

$$
\forall \vec{c}=\{c(k)\} \in \ell^{2}\left(Z^{2}\right) . L c=\sum_{v \in Z^{2}} c(v) T_{v a} \widetilde{f} .
$$

Theorem 1 ${ }^{[5]}$. Let $\left\{T_{v a} f(x)\right\}_{v \in Z^{2}} \subset L^{2}\left(R^{2}\right)$ be a Bessel sequence w.r.t. the subspace $\Omega \subset L^{2}\left(R^{2}\right)$, and $\left\{T_{v a} \widetilde{f}(x)\right\}_{v \in Z^{2}}$ is a Bessel sequence in $L_{2}(R)$. Let $K$ be defined by $(8)$, and $L$ be defined by $(9)$. Assume $P$ is a projection from $L_{2}(R)$ onto $\Omega$. Then $\left\{T_{v} f(x)\right\}_{v \in Z^{2}}$ is an affine pseudo -frame for the subspace $\Omega$ with respect to $\left\{T_{v} \widetilde{f}(x)\right\}_{v \in Z}$ if and only if

$$
K L P=P .
$$

Definition 2. We say that a Generalized multiresolution structure (GMS) $\left\{V_{n}, f(x), \widetilde{f}(x)\right\}$ of the space $L^{2}\left(R^{2}\right)$ is a sequence of closed linear subspaces $\left\{V_{n}\right\}_{n \in Z}$ of $L^{2}\left(R^{2}\right)$ and two elements $f(x), \widetilde{f}(x) \in L^{2}\left(R^{2}\right)$ such that (i) $V_{n} \subset V_{n+1}, \quad n \in Z^{2} ; \quad$ (ii) $\bigcap_{n \in Z} V_{n}=\{0\} ; \bigcup_{n \in Z} V_{n}$ is dense in $L^{2}\left(R^{2}\right)$; (iii) $g(x) \in V_{n}$ if and only if $g(4 x) \in V_{n+1} \quad \forall n \in Z^{2} \quad$ implies $T_{v a} g(x) \in V_{0}$, for any $v \in Z^{2}$; (v) $\left\{T_{v a} f, v \in Z^{2}\right\}$ forms an affine pseudoframe for $V_{0}$ with respect to $\left\{T_{v a} \widetilde{f}\right\}_{v \in Z^{2}}$.

Proposition $1^{[5]}$. Let $\left\{T_{v a} f\right\}$ be an affine pseudoframe for $V_{0}$ w. r. t. $\left\{T_{v a} \widetilde{f}\right\}$. Define $V_{n}$ by

$$
V_{n} \equiv\left\{f(x) \in L_{2}(R): f\left(4^{-n} x\right) \in V_{0}\right\}, \quad n \in Z,
$$

Then, the sequence $\left\{f_{n, v a}\right\}_{v \in Z}$ is an affine pseudoframe for the subspace $V_{n}$ with respect to $\left\{\widetilde{f}_{n, v a}\right\}_{v \in Z}$. Theorem 2. Let $f(x), \widetilde{f}(x) \in L_{2}(R)$ have the properties specified in Proposition 1 such that the co ndition (11) is satisfied. Assume that $V_{n}$ is defined by (11). Then he sequence $\left\{V_{n}, f, \widetilde{f}\right\}$ forms a generalized multiresolution structure.

Proof. We need to prove four axioms in Definition 2. The inclusion $V_{n} \subseteq V_{n+1}$ follows from the fact that $V_{n}$ defined by (11) is equivalent to $P W_{4^{n} \Lambda}$, and $P W_{\Lambda} \subset P W_{4 \Lambda}$. Condition (b) is satisfied .Beca-use the set of all band-limited signals is dense in $L^{2}(R)$. On the other hand, the intersection of all band-limited signals is the trivial function. Condition (c) is an immediate consequence of (11). For

(d), if $\Gamma(x) \in V_{0}, \Gamma(x)=\sum_{v \in Z}\left\langle\Gamma, T_{v a} \widetilde{f}\right\rangle T_{v a} f(x)$. By taking variable substitution, we have for $\forall u \in Z, \Gamma(x-u)=\sum_{v \in Z}\langle\Gamma(\cdot), \widetilde{f}(\cdot-v a)\rangle f(x-v a-u a)=\sum_{v \in Z}\langle\Gamma(\cdot-u a), \widetilde{f}(\cdot-v a)\rangle f(x-v a)$. Or, it is a fact $F\left(T_{v a} \hbar\right)$ has support in $\Omega$ for arbitrary $v \in Z$. Therefore, $T_{v a} f \in V_{0}$. 


$$
\begin{aligned}
& \sum_{m, n \in Z^{d}}\left|\left\langle f(x), D_{a}^{m} H_{n}\right\rangle\right|^{2} \sum_{m, n \in Z^{2}} a^{m}\left|\sum_{k \in Z^{2}} \int_{[(2 k-1) / 2 b,(2 k+1) / 2 b]^{2}} f(x) \bar{H}_{n}\left(a^{m} x\right) d x\right|^{2} \\
= & \sum_{m, n \in Z^{2}} a^{m}\left|\int_{[-1 / 2 b, 1 / 2 b]^{2}} \sum_{k \in Z^{2}} f(x-k / b) \bar{H}_{n}\left(a^{m} x-a^{m} k / b\right) d x\right|^{2} \\
\leq & \sum_{m, n \in Z^{2}} a^{m} / b ! \int_{[-1 / 2 b, 1 / 2 b]^{2}}\left|\sum_{k \in Z^{2}} f(x-k / b) \bar{H}_{n}\left(a^{m} x-a^{m} k / b\right)\right|^{2} d x \\
\leq & \sum_{m, n \in Z^{2}} a^{m} / b ! \int_{[-1 / 2 b, 1 / 2 b]^{2}}\left|\sum_{k, l \in Z^{2}} f(x-k / b) f(x-l / b) H_{n}\left(a^{m} x-a^{m} k / b\right) H_{n}\left(a^{m} x-a^{m} l / b\right)\right| d x \\
= & \sum_{m, n \in Z^{2}} a^{m} / b ! \int_{[((-1-2 k) / 2 b,}\left|\sum_{k, l \in Z^{2}} f(y) f(y+k / b-l / b) H_{n}\left(a^{m} y\right) H_{n}\left(a^{m} y+a^{m} k / b-a^{m} l / b\right)\right| d y \\
= & \sum_{m, n \in Z^{2}} a^{m} / b ! \int_{R^{2}} \sum_{l \in Z^{2}}\left|f(y) f(y-l / b) H_{n}\left(a^{m} y\right) H_{n}\left(a^{m} y-a^{m} l / b\right)\right| d y \\
\leq & \sum_{m, n, l \in Z^{2}} a^{m} / b !\left[\int_{R^{2}}|f(y)|^{2}\left|H_{n}\left(a^{m} y\right) H_{n}\left(a^{m} y-a^{m} l / b\right)\right| d y\right]^{1 / 2}
\end{aligned}
$$

\section{Conclusion}

We characterize the pseudoframes of translates for the subspaces of $L^{2}\left(R^{2}\right)$. The pyramid de composition scheme is derived based on such a GBMS. As a major new constribution the construction of affine frames for $L^{2}\left(R^{2}\right)$ based on a GBMS is presented.

\section{Acknowledgements}

This work was supported by the Natural Science Foundation of Shaanxi Provincial Gover nment (Grant No:2015JM1024) and the Science Research Foundation of Education Department of Shaanxi Provincial Government (Grant No: 12JK0868, 2013JK0564) .

\section{References}

[1] I. Daubechies, A. Grossmann, A. Meyer, Painless nonorthogonal expansions. J. Math. Phys. 1986;27: 1271-1283.

[2] J. J. Benedetto, S. Li, The theory of multiresolution analysis frames and applications to filter banks.Appl. comput. Harmon.Anal. 1998;5: 389-427.

[3] A.Ron, Z. Shen, Affine systems in L2(Rd). (II) Dual systems. J. Fourier Anal. Appl. 1997; 4:617-637.

[4] I. Daubechies, Ten Lectures on Wavelets. SIAM: Philadelphia,1992.

[5] Q. Chen, etal. The characterization of a class of subspace pseudoframes with arbitrary real number translations. Chaos, Solitons \& Fractals. 2009,42(5):2696-2706. 